\title{
Research on Leaf Classification Algorithm Based on the Image Zhiliang Kang ${ }^{1,}$ a and Peng Huang ${ }^{1, b^{*}}$ \\ ${ }^{1}$ College of Mechanical and Electronic Engineering, Sichuan Agricultural University, Ya'an, 625014, China aZhiliangkang96@163.com, ${ }^{b}$ hpsjdyd@qq.com \\ * The Corresponding Author
}

Keywords: Leaf classification; Image processing; Neural network; Support vector machine (SVM)

\begin{abstract}
The MATLAB image processing toolbox is applied to extract 8 classical features of leaf (including perimeter, area, roundness, complexity, elongation, sphericity, average coefficient variation, serration), and 400 leaf samples are classified respectively on BP Neural Network, Probabilistic Neural Network (PNN) and Support Vector Machine (SVM), and the coverage recognition rate for BP Neural Network, PNN and SVM are obtained as $87.22 \%, 88.95 \%$ and $95.15 \%$ respectively. The coverage recognition rate of SVM is the highest and stable, which can effectively prevent the low recognition rate.
\end{abstract}

\section{Introduction}

With the rapid development of computer technology, the application of computer has become increasingly important in all fields. Especially, it has made great research achievements in the analog stimulation of plant growth and has large value in the classification of plant leaves. According to the comprehensive features of plant leaf shape, making classification on leaf by computer technology has solved the problems of applying genetic inheritance on the classification of leaves and has great significance for further research in the law of plant leaves. At present, there are very few researches on the classification of leaves in China. Researchers like Wang Xiaofeng and Hou Tong have proposed the suggestion on the application of the features of leaf shape by making classification on BP Neural Network, however, the main problem is that the recognition rate is not very high, and it is difficult to avoid the low recognition rate as the unstable recognition rate of BP Neural Network [1, 2]. In addition, researchers like He Peng and Huang Lin et al have also proposed the suggestion of applying PNN for the classification of leaves. Although it has higher recognition rate than BP Neural Network, it also faces with unstable and low recognition rate [3, 4]. This essay will introduce SVM into the classification of leaves, as it has a higher and stable recognition rate compared with BP Neural Network and PNN.

\section{Image Preprocessing}

Threshold Segmentation. Threshold segmentation refers to distinguishing the foreground and the background of an image. As the influence of color difference and photograph conditions, it cannot use unified gray threshold for the segmentation on the images of different leaves. It uses the iterative threshold selection method in this essay, and the steps and methods are as follows:

Step1. To calculate the maximum gray value and the minimum gray value of the whole image, and write down as $H_{\max }$ and ${ }_{\text {min }}$, so the initial gray thresholds $\mathrm{t}=\left(\mathrm{H}_{\max }+\mathrm{H}_{\min }\right) / 2$;

Step2. To segment the image into foreground and background by the calculated threshold ${ }_{k}$, and then respectively calculate the average gray values ${ }^{H_{1}}$ and ${ }^{H_{2}}$ of the two parts. 
Step3. To calculate the new threshold $\mathrm{t}_{\mathrm{k}+1}=\left(\mathrm{H}_{1}+\mathrm{H}_{2}\right) / 2$;

Step4. If $t_{k+1}=t_{k}$, so $t_{k}$ is the optimal segmentation threshold, and otherwise back to steps2 to do iterative calculation again.

Binaryzation. After getting the optimal gray threshold, the gray value in the leaf image area is larger or equal to the pixel with the optimal gray threshold, which is written down as 1 , otherwise as 0 .

Morphological Processing. In order to eliminate parts of stains and make the edge more clear, it makes morphological opening operation on the binary image by using the definition structure of function strel in MATLAB image processing toolbox with the operator parameters selection of rectangle.

\section{Feature Extraction}

Normally, leaves image can be extracted a lot of characteristic values, such as rectangularity, axis ratio, convex concave, roundness, eccentricity, serration, bending energy, curvature characteristics, direction angle, best matching ellipse, sphericity, area convexity, perimeter convexity, etc. For tree species diversity, leaves diversity and leaves specific attributes, this essay has extracted 8 characteristic values as the evidence of identifying tree species, including perimeter $\mathrm{L}$, area $\mathrm{S}$, roundness $\mathrm{C}$, complexity e, elongation $\mathrm{E}$, spericity s, average coefficient variation $\mathrm{d}$ and serration $\mathrm{M}$.

This essay has selected 8 classical leaves as experimental samples. Each kind of leaves has 50 samples, and the experimental samples are 400 in total. Fig. 1 is the 8 leaf samples, and 8 characteristic values are extracted from the 8 samples as shown in Table 1.
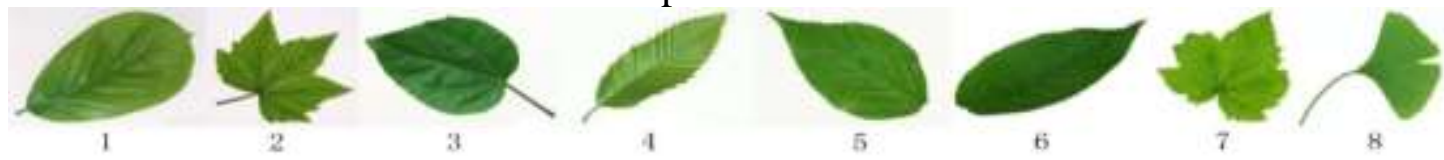

Figure 1. Eight Kinds of Typical Tree Leaves

Table 1 Eight characteristic values from 8 typical tree leaves

\begin{tabular}{|c|c|c|c|c|c|c|c|c|}
\hline $\begin{array}{l}\text { characteristic } \\
\text { values }\end{array}$ & $L$ & $S$ & $C$ & $e$ & $E$ & $S$ & $d$ & $1 / M$ \\
\hline \multirow{2}{*}{1} & & 418 & 0.6 & 17.0 & 0.8 & 0.7 & & \\
\hline & 875.3858 & 77 & 995 & 826 & 175 & 611 & 0.0828 & 145.5803 \\
\hline \multirow{2}{*}{2} & & 274 & 0.2 & 45.1 & 0.8 & 0.4 & & \\
\hline & 1100.1880 & 33 & 646 & 093 & 154 & 840 & 0.0070 & 5.6480 \\
\hline \multirow{2}{*}{3} & & 326 & 0.5 & 25.8 & 0.8 & 0.7 & & \\
\hline & 973.9597 & 53 & 013 & 126 & 034 & 208 & 0.0087 & 27.5117 \\
\hline \multirow{2}{*}{4} & & 211 & 0.3 & 34.9 & 0.7 & 0.6 & & \\
\hline & 789.4935 & 68 & 697 & 106 & 837 & 507 & 0.0243 & 11.8722 \\
\hline \multirow{2}{*}{5} & & 366 & 0.5 & 20.9 & 0.7 & 0.7 & & \\
\hline & 865.4403 & 70 & 560 & 295 & 811 & 434 & 0.0211 & 6.2776 \\
\hline \multirow{2}{*}{6} & & 325 & 0.5 & 21.8 & 0.6 & 0.5 & & \\
\hline & 882.7207 & 40 & 700 & 139 & 406 & 857 & 0.0297 & 6.8274 \\
\hline \multirow{2}{*}{7} & & 287 & 0.4 & 30.1 & 0.7 & 0.5 & & \\
\hline & 977.6689 & 860 & 254 & 708 & 665 & 760 & 0.0056 & 5.9067 \\
\hline \multirow[t]{2}{*}{8} & & 162 & 0.2 & 48.6 & 0.7 & 0.4 & & \\
\hline & 987.5028 & 98 & 476 & 826 & 176 & 180 & 0.0785 & 38.8441 \\
\hline
\end{tabular}




\section{Classification}

Classification of BP Neural Network. BP Neural Network is a kind of multilayer feed forward network trained by error back propagation algorithm, which is one of the most widely used neural network model at present. BP Neural network requires large amounts of training data for learning and stores a lot of input-output model mapping relations. Its learning rule is to use the steepest descent method, by back-propagation network to continuously adjust the weights and thresholds, so the square of the error of the network is minimal.

Leaf has 8 features, in which BP input layer has 8 layers, and hidden layer setting is 9 layers, and output layer is 8 layers. 300 groups are randomly selected from the 400 groups of sampling data as the training set for the training of BP Neural Network. The remaining 100 groups are selected as testing set to do repeated trails 100 times, and the average recognition rate of BP Neural Network is obtained as $87.22 \%$, with the maximum of $95 \%$ and the minimum of $76 \%$.

Classification of PNN. PNN is a kind of feed forward neural network developed from the Radial Basis Function Network, the theoretical basis of which is Minimum Bayesian Risk Criterion (also called Bayesian Decision Theory). As one of radial functions, PNN is suitable for pattern classification.

Learning algorithm steps of PNN are as follows:

Step 1.To confirm the center of radial basis function of hidden layer neuron.Without loss of generality, to set the training sampling input matrix $\mathrm{P}$ and output matrix $\mathrm{T}$ respectively as:

$$
P=\left[\begin{array}{cccc}
p_{11} & p_{12} & \ldots & p_{1 Q} \\
p_{21} & p_{22} & \ldots & p_{2 Q} \\
\ldots & \ldots & & \ldots \\
p_{R 1} & p_{R 2} & \ldots & p_{R Q}
\end{array}\right] \quad T=\left[\begin{array}{cccc}
t_{11} & t_{12} & \ldots & t_{1 Q} \\
t_{21} & t_{22} & \ldots & t_{2 Q} \\
\ldots & \ldots & & \ldots \\
t_{K 1} & t_{K 2} & \ldots & t_{K Q}
\end{array}\right]
$$

In which, $P_{i j}$ is the ith input variable of the jth training samples; $t_{i j}$ is the ith output variable of the $j$ th training samples; $\mathrm{R}$ is the dimension of input variables; $\mathrm{K}$ is the dimension of output variables, corresponding to $\mathrm{K}$ categories; $\mathrm{Q}$ is the number of training set samples. Each neuron in hidden layer corresponds to each training samples, and the center of radial basis function corresponding to $\mathrm{Q}$ hidden layer neurons is $\mathrm{C}=\mathrm{P}$ '.

Step 2.To confirm the threshold of hidden layer neuron. For simplicity, the threshold corresponding to $\mathrm{Q}$ hidden layer neuron is that:

$$
b_{1}=\left[b_{11}, b_{12}, \ldots b_{1 Q}\right]
$$

In the formula $(1), b_{1}=b_{12}=\cdots=b_{1 Q}=0.8326 /$ spread, the spread is the spread velocity of radial basis function.

Step 3. To confirm the weight between hidden layer and output layer. After confirming the center and the threshold of radial basis function of hidden layer neuron, the output of hidden neuron can be calculated by the above formula:

$$
a^{i}=\exp \left(-\left\|C-p_{i}\right\|^{2} b_{1}\right), i=1,2 \ldots Q
$$

In formula (2), $p_{i}=\left[p_{i 1}, p_{i 2}, \ldots p_{i R}\right]^{\prime}$ is vector of the ith training sample.

In PNN, the connection weight $\mathrm{W}$ between hidden layer and output layer is selected as the output matrix of the training set, which is $\mathrm{W}=\mathrm{t}$.

Step 4. To calculate the output of output layer neuron. After confirming the connection weight between hidden layer neuron and output layer neuron, the output of output layer neuron can be calculated by the Figure 6, that is:

$$
\mathrm{n}^{\mathrm{i}}=\mathrm{LW}_{2,1} \mathrm{a}^{\mathrm{i}}, \mathrm{i}=1,2, \ldots \mathrm{Q}
$$




$$
y^{i}=\operatorname{compet}\left(n^{i}\right), i=1,2, \ldots Q
$$

By repeated trials, this essay selects spread=0.25. 300 groups of samples are randomly selected as the training set for the training of PNN, and the remaining 100 groups are selected as testing set to do repeated trails 100 times, and the average recognition rate of PNN is obtained as $88.95 \%$, with the maximum of $95 \%$ and the minimum of $78 \%$.

SVM Classification. The main though of SVM is to set up a hyperplane as decision surface, and maximize the isolation edge between the positive example and negative example, and its generalization error rate is regarded the sum of the training error rate and the item depending on VC (VapnikChervonenkis dimension) as the boundary. In the case of separable mode, the value of SVM is 0 for the former item and minimizes the second item. SVM has its specific attribute of providing good generalization performance of the classification purposes. See Fig. 4 for the basic structure of SVM.

300 groups of samples are randomly selected as the training set for the training of SVM network. The remaining 100 groups are selected as testing set to do repeated trails 100 times, and the average recognition rate of SVM network is obtained as $95.15 \%$, with the maximum of $100 \%$ and the minimum of $91 \%$.

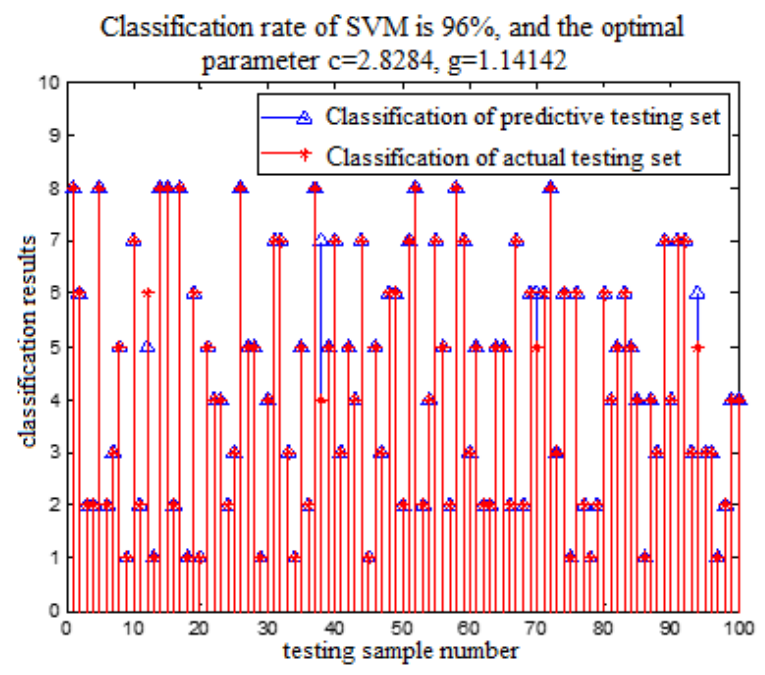

Figure 2. SVM Model Renderings

Comparison of Three Classification Methods.Comparison of recognition rates of the three classification methods show in Table 2. As can be seen from Table 2, the effect of SVM classification is the best in the 400 samples selected in this essay.

Table 2 Comparison of recognition rates of the three classification methods

\begin{tabular}{cccc}
\hline $\begin{array}{c}\text { classification } \\
\text { methods }\end{array}$ & $\begin{array}{c}\text { minimum } \\
\text { recognition rate } \\
(\%)\end{array}$ & $\begin{array}{c}\text { maximum } \\
\text { recognition rate } \\
(\%)\end{array}$ & $\begin{array}{c}\text { average } \\
\text { recognition rate } \\
(\%)\end{array}$ \\
\hline BP & 76 & 95 & 87.22 \\
PNN & 78 & 95 & 88.95 \\
SVM & 91 & 100 & 95.15 \\
\hline
\end{tabular}




\section{Conclusion}

BP network mainly has the problems including unstable recognition rate, slow rate of convergence, easy to fall into local optimal solution, difficult to determine the number of hidden layers, etc. Although PNN network always converges to Bayes optimal solution and its stability is higher than BP, it has a larger value of production and the selection of spread is difficult to determine as well. In addition, even for the same training set and testing set, there also have differences in the recognition rate between BP Neural Network and PNN, which shows that the stability of the two is not good. Unlike BP and PNN, SVM requires small quantity of training samples, and more importantly its recognition rate is a constant for the same training set and testing set, so it has good stability. In term of both recognition and stability, SVM is superior to BP and PNN.

\section{Acknowledgements}

This work was supported by Sichuan agricultural university dual - branch program.

\section{References}

[1] WANG Xiao-feng, et al. Feature Extraction and Recognition for Leaf Images [J] .Journal of Computer Engineering and Applications, 2006,190 193.

[2] Hou tong, Yao Lihong, Kan Jiangming. Plant Recognition Research Based on Shape Features of Leaf [J], Hunan Agricultural Sciences, 2009, (4): 123 125,129.

[3] He Peng, Huang Lin. Feature Extraction and Recognition of Plant Leaf [J]. Journal of Agricultural Mechanization Research, 2008, (6), $168 \sim 170$.

[4] He Peng, et al. Research of Machine Recognition for Broad-Leaved Trees Based on Leaves' Synthetic Features [J]; Northwest A \& F University, 2008, (12).

[5] T W Ridler, S Calvard.Picture Thresholding Using an Iterative Selection Method [J]. IEEE Transaction on System, Man and Cybernetics, 1978; 8 (8): $630 \sim 632$

[6] Ma Xiulian, Yi Shujuan. Based on the embedded system and machine vision of the hatching before the eggless identification system [j], Journal of Agricultural Mechanics, 2011,42 (5), 188 191.

[7] LI Wei, ZHOU Zhao-hui, YAN Cheng-hua. Research on Algorithm for Solving Maximum Inscribed Circle of Image [J] Journal of Engineering Graphics; 2006, (2), 118 120.

[8] Xu Hui, Wang Zhongzhi, Huang Xinyuan. Rapid identification of the toothed leaf margin based on corner detection [J], Journal of Beijing Forestry University, 2010,32 (6), $85 \sim 89$.

[9] Li Yang et al.MATLAB neural network 30 case analysis [M]. Beijing University of Aeronautics and Astronautics Press, 2010,176 182.

[10] $\mathrm{Hu}$ Fei et al. MATLAB intelligent algorithm 30 case analysis $[\mathrm{M}]$. Beijing University of Aeronautics and Astronautics Press, 2011,248 258. 